\title{
Basic Properties of Metrizable Topological Spaces
}

\author{
Karol Pąk \\ Institute of Computer Science \\ University of Białystok \\ Poland
}

\begin{abstract}
Summary. We continue Mizar formalization of general topology according to the book [11] by Engelking. In the article, we present the final theorem of Section 4.1. Namely, the paper includes the formalization of theorems on the correspondence between the cardinalities of the basis and of some open subcover, and a discreet (closed) subspaces, and the weight of that metrizable topological space. We also define Lindelöf spaces and state the above theorem in this special case. We also introduce the concept of separation among two subsets (see [12]).
\end{abstract}

MML identifier: METRIZTS, version: 7.11.02 4.125.1059

The articles [21], [13], [20], [2], [1], [3], [10], [9], [7], [16], [4], [6], [19], [23], [22], [17], [15], [14], [8], [18], and [5] provide the notation and terminology for this paper.

\section{Preliminaries}

For simplicity, we follow the rules: $T, T_{1}, T_{2}$ denote topological spaces, $A, B$ denote subsets of $T, F, G$ denote families of subsets of $T, A_{1}$ denotes a subset of $T_{1}, A_{2}$ denotes a subset of $T_{2}, T_{3}, T_{4}, T_{5}$ denote metrizable topological spaces, $A_{3}, B_{1}$ denote subsets of $T_{3}, F_{1}, G_{1}$ denote families of subsets of $T_{3}, C$ denotes a cardinal number, and $i_{1}$ denotes an infinite cardinal number.

Let us consider $T_{1}, T_{2}, A_{1}, A_{2}$. We say that $A_{1}$ and $A_{2}$ are homeomorphic if and only if:

(Def. 1) $T_{1}\left\lceil A_{1}\right.$ and $T_{2} \uparrow A_{2}$ are homeomorphic. 
Next we state four propositions:

(1) $T_{1}$ and $T_{2}$ are homeomorphic iff $\Omega_{\left(T_{1}\right)}$ and $\Omega_{\left(T_{2}\right)}$ are homeomorphic.

(2) Let $f$ be a function from $T_{1}$ into $T_{2}$. Suppose $f$ is homeomorphism. Let $g$ be a function from $T_{1}\left\lceil A_{1}\right.$ into $T_{2} \uparrow f^{\circ} A_{1}$. If $g=f \uparrow A_{1}$, then $g$ is homeomorphism.

(3) For every function $f$ from $T_{1}$ into $T_{2}$ such that $f$ is homeomorphism holds $A_{1}$ and $f^{\circ} A_{1}$ are homeomorphic.

(4) If $T_{1}$ and $T_{2}$ are homeomorphic, then weight $T_{1}=$ weight $T_{2}$.

Note that every topological space which is empty is also metrizable and every topological space which is metrizable is also $T_{4}$ and non empty. Let $M$ be a metric space. Note that $M_{\text {top }}$ is metrizable.

Let us consider $T_{3}, A_{3}$. Observe that $T_{3}\left\lceil A_{3}\right.$ is metrizable.

Let us consider $T_{4}, T_{5}$. Observe that $T_{4} \times T_{5}$ is metrizable.

Next we state two propositions:

(5) weight $T_{1} \times T_{2} \subseteq$ weight $T_{1} \cdot$ weight $T_{2}$.

(6) If $T_{1}$ is non empty and $T_{2}$ is non empty, then weight $T_{1} \subseteq$ weight $T_{1} \times T_{2}$ and weight $T_{2} \subseteq$ weight $T_{1} \times T_{2}$.

Let $T_{1}, T_{2}$ be second-countable topological spaces. One can check that $T_{1} \times$ $T_{2}$ is second-countable.

One can prove the following propositions:

(7) $\operatorname{Card}(F\lceil A) \subseteq \operatorname{Card} F$.

(8) For every basis $B_{2}$ of $T$ holds $B_{2}\lceil A$ is a basis of $T\lceil A$.

Let $T$ be a second-countable topological space and let $A$ be a subset of $T$. Note that $T\lceil A$ is second-countable.

Let $M$ be a non empty metric space and let $A$ be a non empty subset of $M_{\text {top }}$. One can check that $\operatorname{dist}_{\min }(A)$ is continuous.

We now state the proposition

(9) For every subset $B$ of $T$ and for every subset $F$ of $T\lceil A$ such that $F=B$ holds $T\lceil A \uparrow F=T\lceil B$.

Let us consider $T_{3}$. Observe that every subset of $T_{3}$ which is open is also $F_{\sigma}$ and every subset of $T_{3}$ which is closed is also $G_{\delta}$.

The following propositions are true:

(10) For every subset $F$ of $T\left\lceil B\right.$ such that $A$ is $F_{\sigma}$ and $F=A \cap B$ holds $F$ is $F_{\sigma}$.

(11) For every subset $F$ of $T\left\lceil B\right.$ such that $A$ is $G_{\delta}$ and $F=A \cap B$ holds $F$ is $G_{\delta}$.

(12) If $T$ is a $T_{1}$ space and $A$ is discrete, then $A$ is an open subset of $T\lceil\bar{A}$.

(13) Let given $T$. Suppose that for every $F$ such that $F$ is open and a cover of $T$ there exists $G$ such that $G \subseteq F$ and $G$ is a cover of $T$ and Card $G \subseteq C$. 
Let given $A$. If $A$ is closed and discrete, then Card $A \subseteq C$.

(14) Let given $T_{3}$. Suppose that for every $A_{3}$ such that $A_{3}$ is closed and discrete holds Card $A_{3} \subseteq i_{1}$. Let given $A_{3}$. If $A_{3}$ is discrete, then Card $A_{3} \subseteq$ $i_{1}$.

(15) Let given $T$. Suppose that for every $A$ such that $A$ is discrete holds Card $A \subseteq C$. Let given $F$. Suppose $F$ is open and $\emptyset \notin F$ and for all $A, B$ such that $A, B \in F$ and $A \neq B$ holds $A$ misses $B$. Then Card $F \subseteq C$.

(16) For every $F$ such that $F$ is a cover of $T$ there exists $G$ such that $G \subseteq F$ and $G$ is a cover of $T$ and $\operatorname{Card} G \subseteq \operatorname{Card}\left(\Omega_{T}\right)$.

(17) If $A_{3}$ is dense, then weight $T_{3} \subseteq \operatorname{Card} \omega \cdot \operatorname{Card} A_{3}$.

\section{Main Properties}

Next we state several propositions:

(18) weight $T_{3} \subseteq i_{1}$ if and only if for every $F_{1}$ such that $F_{1}$ is open and a cover of $T_{3}$ there exists $G_{1}$ such that $G_{1} \subseteq F_{1}$ and $G_{1}$ is a cover of $T_{3}$ and Card $G_{1} \subseteq i_{1}$.

(19) weight $T_{3} \subseteq i_{1}$ iff for every $A_{3}$ such that $A_{3}$ is closed and discrete holds Card $A_{3} \subseteq i_{1}$.

(20) weight $T_{3} \subseteq i_{1}$ iff for every $A_{3}$ such that $A_{3}$ is discrete holds Card $A_{3} \subseteq i_{1}$.

(21) weight $T_{3} \subseteq i_{1}$ if and only if for every $F_{1}$ such that $F_{1}$ is open and $\emptyset \notin F_{1}$ and for all $A_{3}, B_{1}$ such that $A_{3}, B_{1} \in F_{1}$ and $A_{3} \neq B_{1}$ holds $A_{3}$ misses $B_{1}$ holds Card $F_{1} \subseteq i_{1}$.

(22) weight $T_{3} \subseteq i_{1}$ iff density $T_{3} \subseteq i_{1}$.

(23) Let $B$ be a basis of $T_{3}$. Suppose that for every $F_{1}$ such that $F_{1}$ is open and a cover of $T_{3}$ there exists $G_{1}$ such that $G_{1} \subseteq F_{1}$ and $G_{1}$ is a cover of $T_{3}$ and Card $G_{1} \subseteq i_{1}$. Then there exists a basis $u_{1}$ of $T_{3}$ such that $u_{1} \subseteq B$ and Card $u_{1} \subseteq i_{1}$.

\section{Properties of Lindelöf SPACES}

Let us consider $T$. We say that $T$ is Lindelöf if and only if:

(Def. 2) For every $F$ such that $F$ is open and a cover of $T$ there exists $G$ such that $G \subseteq F$ and $G$ is a cover of $T$ and countable.

Next we state the proposition

(24) For every basis $B$ of $T_{3}$ such that $T_{3}$ is Lindelöf there exists a basis $B^{\prime}$ of $T_{3}$ such that $B^{\prime} \subseteq B$ and $B^{\prime}$ is countable.

Let us observe that every metrizable topological space which is Lindelöf is also second-countable. 
Let us note that every metrizable topological space which is Lindelöf is also separable and every metrizable topological space which is separable is also Lindelöf.

One can verify the following observations:

* there exists a non empty topological space which is Lindelöf and metrizable,

* every topological space which is second-countable is also Lindelöf,

* every topological space which is $T_{3}$ and Lindelöf is also $T_{4}$, and

* every topological space which is countable is also Lindelöf.

Let $n$ be a natural number. Note that the topological structure of $\mathcal{E}_{\mathrm{T}}^{n}$ is second-countable.

Let $T$ be a Lindelöf topological space and let $A$ be a closed subset of $T$. One can verify that $T\lceil A$ is Lindelöf.

Let $T_{3}$ be a Lindelöf metrizable topological space and let $A$ be a subset of $T_{3}$. One can verify that $T_{3} \uparrow A$ is Lindelöf.

Let us consider $T$ and let $A, B, L$ be subsets of $T$. We say that $L$ separates $A, B$ if and only if:

(Def. 3) There exist open subsets $U, W$ of $T$ such that $A \subseteq U$ and $B \subseteq W$ and $U$ misses $W$ and $L=(U \cup W)^{\mathrm{c}}$.

The following two propositions are true:

(25) If $A_{3}$ and $B_{1}$ are separated, then there exists a subset $L$ of $T_{3}$ such that $L$ separates $A_{3}, B_{1}$.

(26) Let $M$ be a subset of $T_{3}, A_{1}, A_{2}$ be closed subsets of $T_{3}$, and $V_{1}, V_{2}$ be open subsets of $T_{3}$. Suppose $A_{1} \subseteq V_{1}$ and $A_{2} \subseteq V_{2}$ and $\overline{V_{1}}$ misses $\overline{V_{2}}$. Let $m_{1}, m_{2}, m_{3}$ be subsets of $T_{3}\left\lceil M\right.$. Suppose $m_{1}=M \cap \overline{V_{1}}$ and $m_{2}=M \cap \overline{V_{2}}$ and $m_{3}$ separates $m_{1}, m_{2}$. Then there exists a subset $L$ of $T_{3}$ such that $L$ separates $A_{1}, A_{2}$ and $M \cap L \subseteq m_{3}$.

\section{REFERENCES}

[1] Grzegorz Bancerek. Cardinal arithmetics. Formalized Mathematics, 1(3):543-547, 1990

[2] Grzegorz Bancerek. Cardinal numbers. Formalized Mathematics, 1(2):377-382, 1990.

[3] Grzegorz Bancerek. König's theorem. Formalized Mathematics, 1(3):589-593, 1990.

[4] Grzegorz Bancerek. The ordinal numbers. Formalized Mathematics, 1(1):91-96, 1990.

[5] Józef Białas and Yatsuka Nakamura. The theorem of Weierstrass. Formalized Mathematics, 5(3):353-359, 1996.

[6] Leszek Borys. Paracompact and metrizable spaces. Formalized Mathematics, 2(4):481485, 1991.

[7] Czesław Byliński. Functions from a set to a set. Formalized Mathematics, 1(1):153-164, 1990.

[8] Agata Darmochwał. Families of subsets, subspaces and mappings in topological spaces. Formalized Mathematics, 1(2):257-261, 1990.

[9] Agata Darmochwał. Finite sets. Formalized Mathematics, 1(1):165-167, 1990.

[10] Agata Darmochwał. The Euclidean space. Formalized Mathematics, 2(4):599-603, 1991.

[11] Ryszard Engelking. General Topology, volume 60 of Monografie Matematyczne. PWN Polish Scientific Publishers, Warsaw, 1977. 
[12] Ryszard Engelking. Teoria wymiaru. PWN, 1981.

[13] Adam Grabowski. Properties of the product of compact topological spaces. Formalized Mathematics, 8(1):55-59, 1999.

[14] Adam Grabowski. On the Borel families of subsets of topological spaces. Formalized Mathematics, 13(4):453-461, 2005.

[15] Adam Grabowski. On the boundary and derivative of a set. Formalized Mathematics, 13(1):139-146, 2005.

[16] Stanisława Kanas, Adam Lecko, and Mariusz Startek. Metric spaces. Formalized Mathematics, 1(3):607-610, 1990.

[17] Zbigniew Karno. Maximal discrete subspaces of almost discrete topological spaces. Formalized Mathematics, 4(1):125-135, 1993.

[18] Robert Milewski. Bases of continuous lattices. Formalized Mathematics, 7(2):285-294, 1998.

[19] Beata Padlewska and Agata Darmochwał. Topological spaces and continuous functions. Formalized Mathematics, 1(1):223-230, 1990.

[20] Alexander Yu. Shibakov and Andrzej Trybulec. The Cantor set. Formalized Mathematics, 5(2):233-236, 1996.

[21] Andrzej Trybulec. A Borsuk theorem on homotopy types. Formalized Mathematics, 2(4):535-545, 1991.

[22] Zinaida Trybulec. Properties of subsets. Formalized Mathematics, 1(1):67-71, 1990.

[23] Edmund Woronowicz. Relations defined on sets. Formalized Mathematics, 1(1):181-186, 1990.

Received March 31, 2009 